\title{
The Personal Interaction Panel - a Two-Handed Interface for Augmented Reality
}

\author{
Zsolt Szalavári and Michael Gervautz \\ Institute of Computer Graphics, Vienna University of Technology \\ Karlsplatz 13/186/2, A-1040 Vienna, Austria \\ \{szalavari,gervautz\}@cg.tuwien.ac.at - http://www.cg.tuwien.ac.at/
}

\begin{abstract}
This paper describes the introduction of a new interaction paradigm to augmented reality applications. The everyday tool handling experience of working with pen and notebooks is extended to create a three dimensional two-handed interface, that supports easy-to-understand manipulation tasks in augmented and virtual environments. In the design step we take advantage from the freedom, given by our very low demands on hardware and augment form and functionality to this device. On the basis of examples from object manipulation, augmented research environments and scientific visualization we show the generality of applicability. Although being in the first stages implementation, we consider the wide spectrum of suitability for different purposes.
\end{abstract}

Keywords: 3D user interface, augmented reality, two handed interaction.

\section{Introduction}

Despite the extraordinary rapid development of computers and software for virtual reality, the acceptance of full immersive systems follows these growth rates rather hard. The aim to create the feeling of immersion by presenting convincing stimuli to the user is still not really satisfying, we are far from high-fidelity in virtual reality. Especially social aspects of full immersion may play a substantial role in denying usage by some people.

Augmented reality (AR) offers a smooth immersion by leaving the connection to real-world environments in principle untouched and superimposing computer generated imagery onto real artefacts. Social communication channels as natural speech and paralanguage are not blocked, breaking down mental barriers of applying virtual reality technology to a specific problem. Not unexpectedly, a lot of different problems arise in the investigation of augmented reality, like registration and occlusion of real world obstacles, but much research is concentrating on these topics [Baju95],[Stat96].

Another critical part of these systems lies in the interaction methods provided to the user. Whereas conventional desktop input devices, e.g. keyboard and mouse have reached a high degree of specialization during a synthetic evolutionary process, 3D input devices have still significant disadvantages. High accuracy mechanical devices are somewhat bulky or bound to certain applications and do not support generalized interaction 
techniques. Six degree-of-freedom mice and data gloves or suites extend the possible set of interactions by adding nearly unconstrained three dimensional movement and capturing dozens of position and orientation data, but suffer either from unsatisfying low interaction bandwidth or an overloaded metaphor like complex gesture languages. Since no direct tactile feedback of the virtual objects is provided in most systems, inexperienced users feel disoriented first and find it rather difficult to work with flying buttons, menus, "3D widgets" [Conn92] and similar metaphors floating around them.

We introduce a two-handed input device that allows users to use everyday knowledge about interaction, so that no special metaphors are needed and a multitude of interaction styles is supported. Our pair of magnetic tracked pen and pad gives sufficient tactile feedback and is familiar to inexperienced individuals, enabling them concentration on the tasks to be performed. Our implementation with see-through head-mounted displays augments synthesized imagery onto real world objects, but as illustrated in the later sections - this interaction technique serves well in both virtual and augmented environments. Rather than offering virtual devices for manipulation tasks we propose "extended devices“. Extending the real world tools by added virtual shape and functionality while preserving parts of the tool being always identical [Wlok95], users feel to hold all tools in their hands. The simple hardware setup gives much freedom on designing different impressions to fit a wide range of applications from basic object manipulation to complex steering and investigation tasks for scientific visualization.

\section{Related Work}

Sachs et. al. show in their paper [Sach91] how insufficient sophisticated CAD tools serve shape design. Being unable to rough out initial ideas directly with these interaction techniques, designers prefer to use pen and paper instead. A sufficiently intuitive and easy to use approach of "design directly in 3D", using two hand-held six-degree-offreedom sensors in form of a stylus and palette has been proposed. The palette is used to define a reference frame to which objects being drawn are attached, giving the possibility of moving the object instantly into the right angle for viewing on the monitor. While giving the palette a secondary problem, the pen is employed to draw and edit freeform curves directly in 3D. However, the system was not an immersive and head-tracked application, it was suitable for CAD shape design.

A different problem is addressed by Pausch et. al. [Paus95],[Stoa95]. Navigation in immersed virtual environments may become a difficult task for some users, since adaptation to new metaphors requires an introductory phase. In addition to the first person view the developer of the World in Miniature (WIM) metaphor supply a God's eye view of the life-sized surrounding space on a hand-held clipboard. Navigation, locomotion and object manipulation can be achieved at different scales by directly manipulating objects on the WIM and getting feedback on the scale of immersion. Edwards and Hand [Edwa97] describe similar approaches in their work about the prototype of their user interface MaPS for navigation planning and viewpoint manipulation, which they implemented as an extension to immersive VRML2 [Hart96] browsers. In their implementation the user interface is part of the virtual environment itself and supports a wide variety of navigation metaphors.

Even though no pen and paper paradigm is used in their implementation, Amselem [Amse95], Fitzmaurice [Frit93] and Rekimoto [Reki95] show a one-handed interaction metaphor which resembles various aspects of our work. They open a window on a shared virtual environment by giving the user hand-held display (HHD) devices to explore a parallel-world behind reality. The magnetic tracked display can be carried around, showing situated graphics augmented over the real environment. User interaction covers mainly a browsing task of large, spatially distributed, multi-media databases, but implementations also include features, like zooming, layers and browsing of remote environments.

Goble [Gob195], Kabbash [Kabb94] and many other research groups evaluated two-handed interaction as being suitable for a bunch of applications. Their conclusions imply that bimanual interaction can rise overall performance, especially in cases where asymmetric division of labour is applied to the 
hands. Nevertheless the application of a bimanual interface for a specific task needs careful analysis, since the enriched interface may possibly degrade the quality of interaction in some cases.

\section{The Personal Interaction Panel}

To represent the suitability of our tool for a wide range of interaction styles we called our hardware setup Personal Interaction Panel (PIP). The PIP is composed of a lightweight, notebook-sized handheld panel and a pen. Both panel and pen are tracked in position and orientation either by standard magnetic trackers or by optical tracking. We designed concepts for different implementation levels of the panel and pen pair, depending on the supporting software and hardware environment:

- Pressure-sensitive flat display with pen observed with LCD shutter glasses: This setup resembles hardware as shown in [Mel88] and [Weis91] and an enhanced version of today's commercially available hand-held palmtop computers like the Apple Newton Message Pad. Using LCD shutter glasses, the continuously updated computer imagery on the display shows three dimensional images appearing to escape from the flat display, floating above it's surface as if it would be a portable "Responsive Workbench" [Krüg95]. Being portable the display offers exploration of an augmented environment as proposed in [Amse95] and [Reki95] too. The pressuresensitive surface allows not only click-actions, but also fine-grained sensing of pen actions which can improve the interaction capabilities. The disadvantage of this technology is besides the demanding hardware, the limitation by a relatively steep viewing angle, due to the display technology used.

- $\quad$ Pressure-sensitive flat panel with pen and a seethrough head mounted display: All benefits from using a pressure-sensitive device remain in this case, only the display surface has moved from the surface of the panel to the eyes. Synthetic imagery is displayed at real time to users in a see-trough head-mounted display (HMD). The level of augmentation and immersion increases, since users without
HMDs do not see anything on the board, yet they recognize the panel being an input device supporting computer-human interaction. Furthermore a HMD system allows not only projection on a panel but extends augmentation into the whole environment. A drawback of this system is, that full interaction is limited to a specific part of the panel.

- "Dumb" panel and pen in combination with a see-through head mounted display: Neither pad, nor pen have any built-in hardware intelligence apart from the trackers mounted on them, similar to all other versions above. The three dimensional imagery is presented to the user on a see-through HMD, in accordance to his actual viewpoint and viewing direction. Position and orientation tracking of all three parts (panel, pen and HMD) allows the correct evaluation of spatial relations for perspective matching of the real and augmented environment. The physical properties of the devices support exclusively tactile feedback to the user, enriching the interaction.

\subsection{The "dumb" panel}

Although being technically plausible the first variant requires extensive hardware development which would implement our ideas hardwired. Furthermore we see the viewing angle constraint to be very hindering. Whereas a simple selection signal for a pick event on the panel, coming directly from the hardware would be helpful, the second variant has several limitations: the sensitive area is fixed in it's size and properties, most pressure sensitive panels are to massive for continuous hand-held use and the device interferes with state of the art magnetic tracking systems. For our implementation we used the third variant, offering the widest spectrum of degrees of freedom in designing the interface itself and being the most flexible for rapid prototyping, as it has hardly any hardware limitations and software design determines the full functionality.

The PIP interface reaffirms previous results in bimanual action [Guia87], [Hinc94b], [Kabb94] and [Sach91]. The panel defines a base in three dimensional space with the non-dominant hand and 
determines the frame of action of the dominant hand. Users first position the pad and do the finer interaction after that with their dominant hand, as the cognitive load of coarse and fine tasks is asymmetric. Thus a supporting performance of both hands was observed, rather than parallel action. Our interface is well suited for both right- and lefthanded users, a system design issue was, not to incorporate preferences. Test persons did not report problems of fatigue. They immediately realized that wearing a HMD allows to change the point of view rather quickly, so lowering the arm, using the PIP sitting or even placing it for a short time on a desk does not have an impact on its use.

Coincidence between real props and virtual representation of the tools made it natural to lay down and find again the tools, even in the environment of our crowded lab. Despite our implementations gather around augmented reality applications, most of the Personal Interaction Panels functionality can be translated to virtual environments. Since the "dumb" panel does not require to be seen, full immersion of the user into a virtual environment is also possible. Tactile properties of the interface device are saved and coherence in shape of the real tool and the virtual tool helps satisfying the need for convincing stimuli like also shown in [Hinc94b], [Wlok95].

Our combination of an interface with an empty flat surface and sophisticated see-through HMD display technology allows the projection of three dimensional imagery everywhere around the user, but gives also an opportunity to recycle 2D GUI elements. The Personal Interaction Panel supports this mixture of the 2D desktop metaphor and the 3D display, so 2D interaction and three dimensional direct manipulation are done in parallel. Unlike many other interfaces it implements a $2 \mathrm{D}$ interface in 3D, like a notebook with its flat surface in the real world, rather than a combination of $2 \mathrm{D}$ and $3 \mathrm{D}$, requiring a mental change from flat to spatial.

This means in detail that a conventional 2D computer display can be projected onto the board, supporting the 2D desktop metaphor better than "flying menus" or buttons [Butt92], [Harm96], [Jaco93]. Having the tactile feedback of the surface and the fine grained interaction of one hand in the frame of action of the other hand, users can easily interact with desktop elements. Note, the interface does not float around in space, because it is still "directly" connected to the users hand. This imitates holding it in the hand. Thus locating user interface elements becomes rather easy. Dialog boxes for the manipulation of parameters are laid onto the panel and selection or generation of other events is done with the pen. In addition to all these $2 \mathrm{D}$ user interface elements 3D user interface elements such as $3 \mathrm{D}$ widgets are incorporated in the interface of the PIP, supporting three dimensional tasks better than 2D elements. Rising the tip of the pen from the surface of the panel makes it to a six-degree of freedom manipulation device for direct manipulation or selection, in combination with the panel or without it, similar to real world pens, which we use for pointing or as an aid to envision other objects in discussions and the 'reach in with a hand-held stylus' metaphor described in [Serr95]. Again, the 2D in 3D axiom makes this mixture natural and no cognitive switch is necessary.

\section{Interaction using the PIP interface}

As the PIP is a multi-purpose interaction device, the wide range of applications has to be categorized for further investigation. We categorized user interaction tasks in object manipulation, navigation and system control respectively. We will follow this classification and show the usability of the PIP to certain tasks, as well as it's advantages compared to other tools.

\subsection{Object Manipulation}

Modelling of objects has been an issue from the beginning of computer-human interaction. Working with objects directly in three dimensions rather than with 2D projections improves understanding of shape and relations. In augmented environments one has the possibility to compare a real model with the modelled copy of it. Overlaying of real and virtual model or extending a real object with virtual parts (e.g., seeing ultrasound imagery in the patient [Baju92]) or annotating real world objects [Rose95] employ the real capabilities of AR.

Basic object manipulation tasks, like object selection, transformation and on a higher level 
dragging and dropping of objects in three dimensions have been investigated by many research groups [Butt92], [Conn92]. In our setup, the pen alone is used for 3D pointing-, selection operations or direct manipulation of the displayed model, where a 6D mouse is normally used. This feature is seamlessly integrated within the extended PIP functionality, so that the PIP supports a superset of "standard" 3D operations in virtual and augmented reality.

Furthermore selection of objects floating in the augmented scene is enriched by showing the selected item simultaneously on the panel (Figure 1). The contemporary display permits adding additional information to the selection (e.g., physical properties like volume, etc.) on the panel.

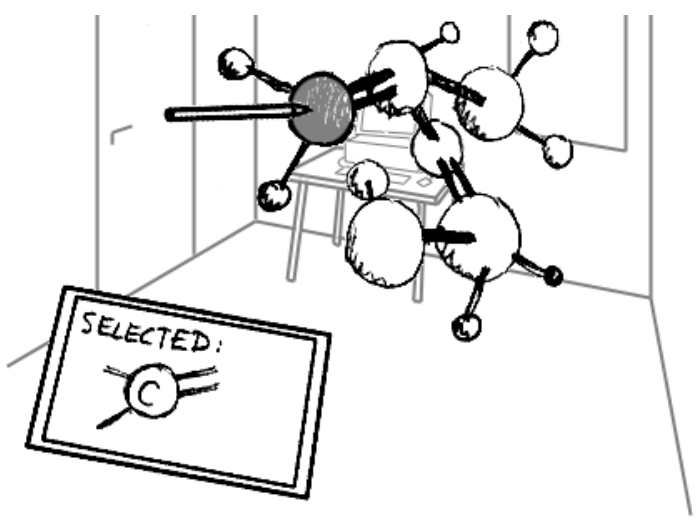

Figure 1. Direct selection of objects by inserting the pen into the "floating" model (background illustrates augmentation)

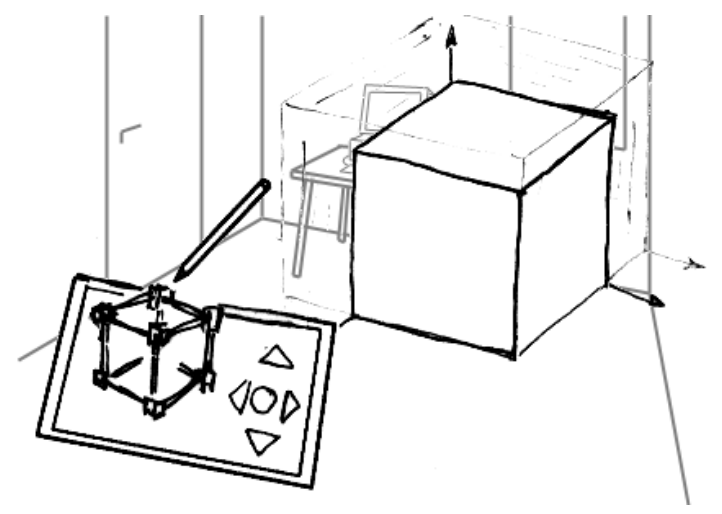

Figure 3. In addition to direct manipulation, widgets can be used for exact scaling ...
The PIP is capable to be used as a visible 3D clipboard carrying a collection of 3D (or 2D) data items, that are shown above the panel's surface and can freely be accessed by the user. Objects may be dragged out from the surface of the PIP and directly placed or moved in the augmentation (Figure 2). This mechanism gives a natural interactive feeling of handling spatial aligned data. Once more the tactile feedback supports interaction, giving the user the feeling, to hold the items in the hand. Correct placement of the objects on the surface, allows to picking on the panel, as if force feedback would have been added.

Besides direct manipulation, handling objects with abstract metaphors like 3D widgets can be useful for certain applications. Unlike many other works,

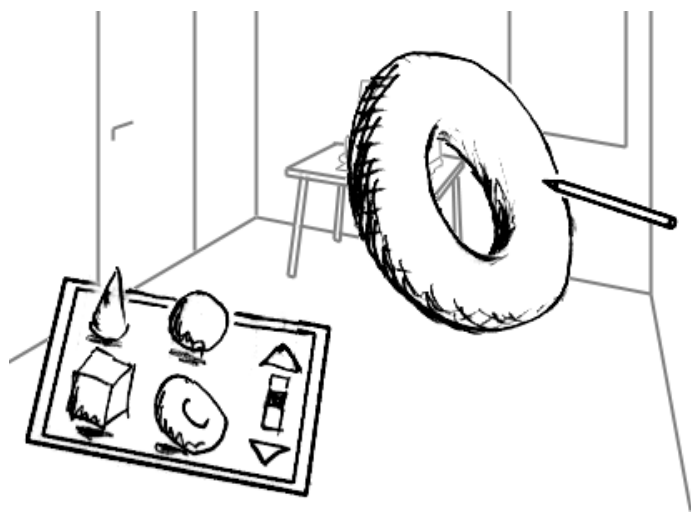

Figure 2. "Drag \& Drop" objects from a clipboard in 3 dimensions

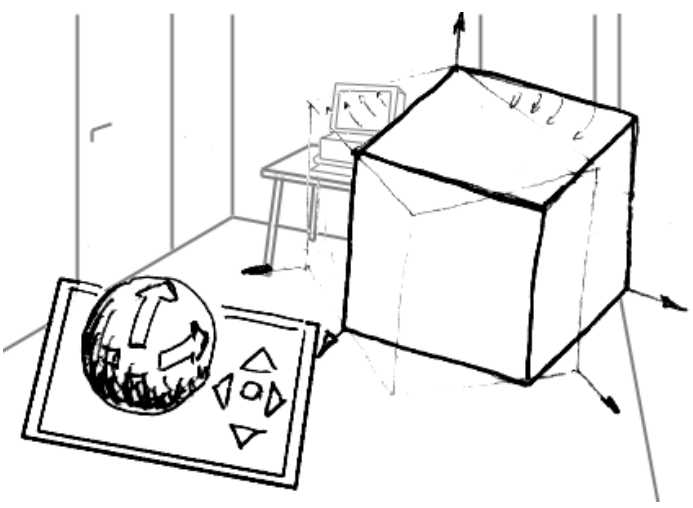

Figure 4. ... or rotation of objects 
the manipulation draggers are in our case not attached to the object but to the surface of the PIP, enabled by the mixture of $2 \mathrm{D}$ and $3 \mathrm{D}$ desktop elements, described above. Advantages of twohanded interaction and in particular the PIP, like frame of reference and panel attached interface elements, improve work with these abstract tools (Figure 3 and 4).

A 3D tool-palette in Figure 5, comparable to the object browser clipboard in Figure 2, contains all basic functionality of the PIP. Among others features like cut and paste tools, "Magic Lenses" [Bier93] and coloring brushes are supported. Attaching these augmented tools to the tip of the pen like shown in [Serr95], the user is given the feeling of holding an "extended" tool in his or her hand.

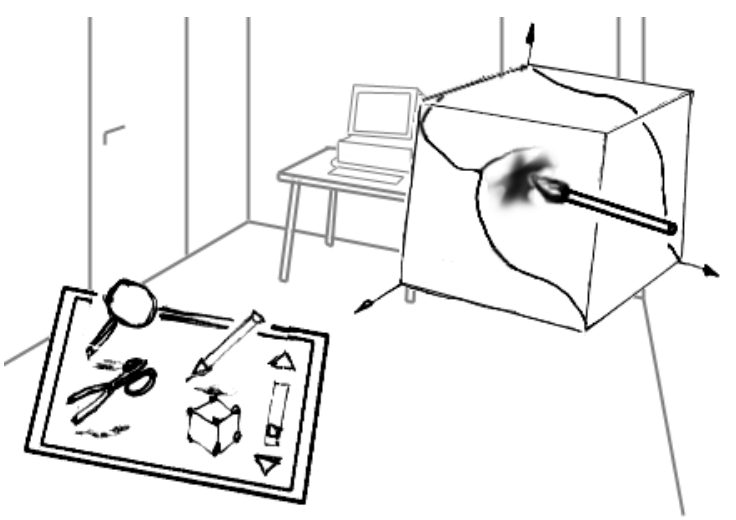

Figure 5. General PIP tools (colouring brush)

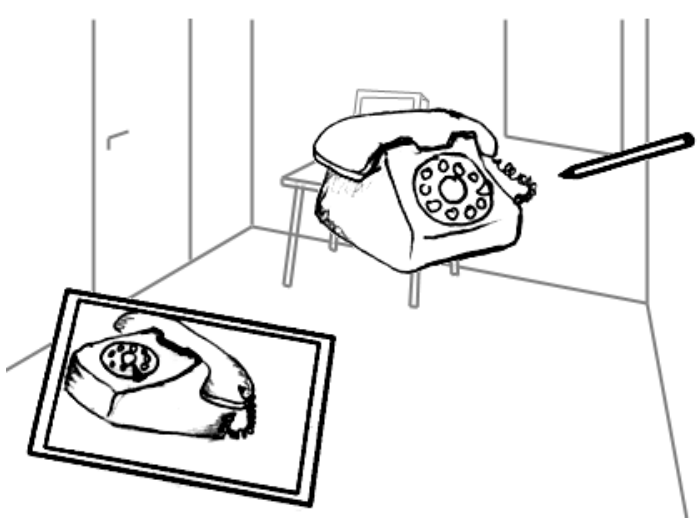

Figure 6. Camera positioning with the pen

\subsection{Navigation}

As object manipulation concentrates on handling and editing of objects, navigation is necessary for changing viewpoint position and orientation in order to explore a specific part of the environment. In our augmented reality setup the user is wearing a HMD, 6D-tracked with magnetic sensors. Movement of the head corresponds automatically to a change of the viewpoint, thus coincidence between real and virtual imagery is ensured.

Additional to the own viewpoint movement, navigation metaphors described in [Hinc94a] are supported in our AR setup with the PIP interface. The "eyeball-in-hand" metaphor is in our case a "look where you point" image, for that virtual camera position and orientation is defined with the pen, while the off-screen rendered camera image is displayed on the panel, as shown in Figure 6. The "scene-in-hand" metaphor similar to the WIM interface [Paus95] and [Stoa95] and the MaPS interface in [Edwa97] can be supported, showing an overview of the surrounding environment.

Not only one-to-one mapping or miniaturized overviews, but magnified details, even from the inside of a simulated object, enhance working in this environment and are supported by the PIP. Figure 7 shows a zoomed detail on the PIP, while observation of the whole object is still possible in parallel.

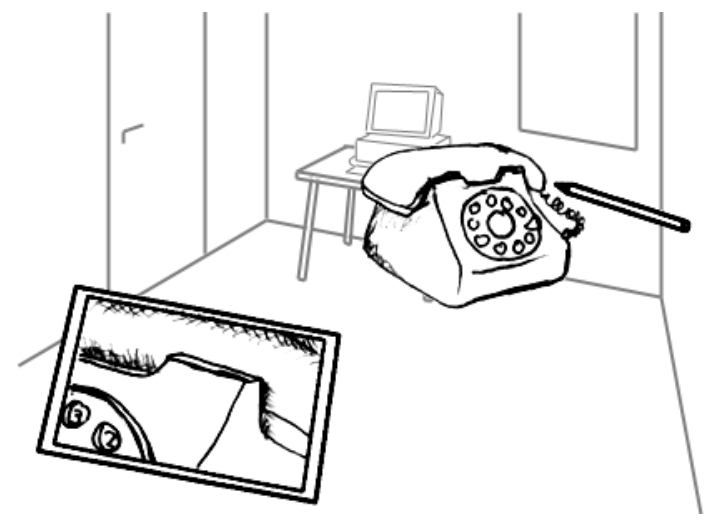

Figure 7. Enlarged view of a detail 
During navigation the panel is possible to act as a virtual photo camera to take a "snapshot" of the scene's state. The currently displayed image or detail is immediately frozen on the panel's surface and e.g., printed out on a remote printer.

It is very important to summarize, that all navigation metaphors presented here in combination with the Personal Interaction Panel have one in common, while using whatever metaphor for navigation with the PIP, the users still keep the connection to the environment and don't have to make a cognitive switch, because the own view is kept and the navigation display is on the panel.

Considering that AR applications need the correspondence between real and virtual environment, navigation and locomotion independent from the real viewpoint is not used, as it would destroy the augmentation. However, for specific applications the PIP is able to support further navigation metaphors in the future, like specifying direction of movement with the pen or "spaceship" control gadgets (2D buttons or 3D widgets) on the panel's surface. These controls mainly support VR applications.

\subsection{System Control}

The design of overall controls is crucial for a system, the user may not be forced to need very special skills for the general controls of the application, as he wants to concentrate on the topic of the application. Immersive augmented applications need easy controls, which have to be "inside" the augmentation. For the feeling of good immersion it is very important not to leave and join the augmentation for operations like reconfiguration of the system, starting a new session, etc. Many solutions transfer conventional application controls to AR or VR systems, like flying menus [Butt92], [Harm96] which have drawbacks, not considering the three dimensional behaviour of this applications, the reduced target acquisition skills in space and the lack of tactile feedback [Hinc94a]. Other examples attach menus to the interface [Wlok95], so target acquisition has only to be relative, enhancing performance.
The PIP offers the possibility to contain and manipulate all the necessary controls in a desktop manner as described above and shown in Figure 8. A tool-palette (Figure 5) groups functions and make them easily accessible.

\subsection{Privacy and Publicity}

We intend to use the PIP interface in a multi-user situation, where it is crucial that the Personal Interaction Panel becomes really personal. Multiple users collaborating in one augmented environment may communicate their ideas and share information. As described below, we are currently working on a project to realize such a system for augmented scientific visualization.

The aspect of allowing users to keep individual information on their PIPs implicates the sharing or concealing of this information, while the overall appearance of the augmentation should stay untouched. One way to build a bridge between private and public is to place "projection walls" into the environment. These walls are static, virtual objects in the augmented environment and are logically connected to one user's PIP, reflecting all changes immediately to the public. Thereby, a single user interaction tool is transformed into a multi-user presentation spot, where personal ideas can be shared.

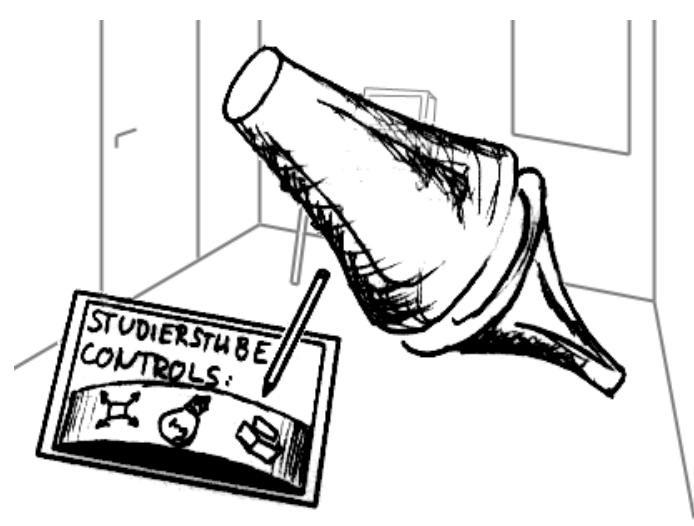

Figure 8. Controlling general parameters of an application, such as system setup, etc. 
Another way to share ideas is to allow "giving" the content of one's hand-held flat PIP to other users. This is achieved with the extension of the drag and drop mechanism to the third dimension, the whole contents of one PIP may be logically moved to another users interaction panel.

All augmented information is automatically hidden for not immersed users. Participants in the immersed environment may see public objects standing around them, but information on other users PIP should be possibly hidden from them. As every user wears a see-through HMD on his own and thus imagery is rendered for every user individually, filtering of parts of the scene, e.g. information on other users PIP, is easily achieved.

\subsection{Implementation}

In our existing implementation pad and pen are tracked with Polhemus Fastrak six degree-offreedom tracking sensors. These are connected to a tracker server, which is implemented on a dedicated workstation.

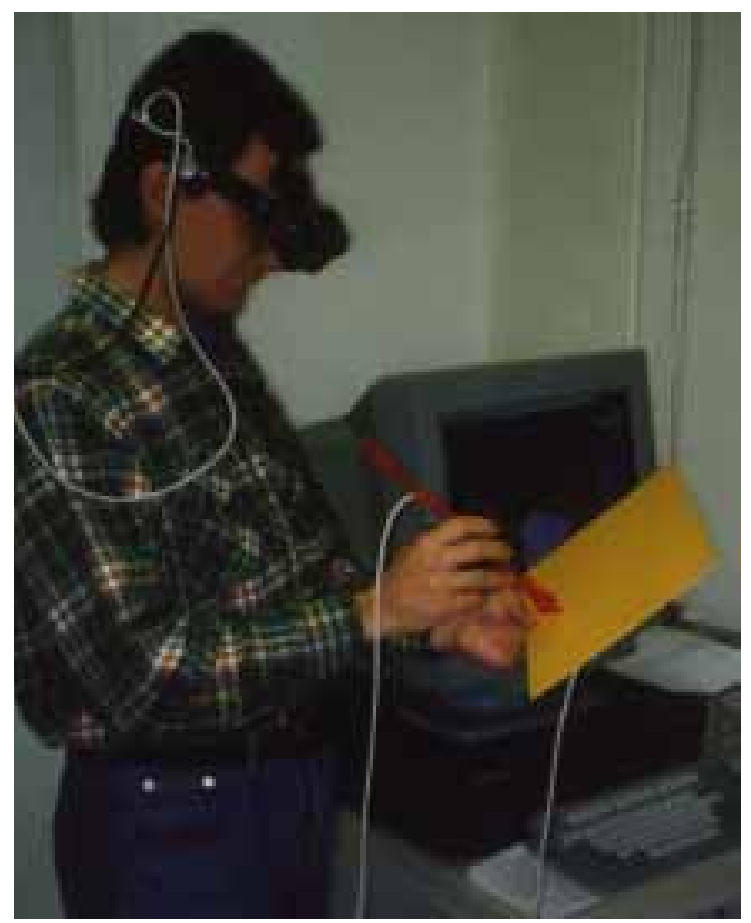

Figure 9. The physical setup of the Personal Interaction Panel during work
The tracker sever sends position and orientation data with TCP/IP protocols over an Ethernet network connection to the rendering system. All three dimensional rendering is done on a Silicon Graphics Indigo2 Maximum Impact computer using Inventor graphics libraries. The resulting image is superimposed onto the real environment using a Virtual I/O i-Glasses! head-mounted display. The fast rendering hardware allows high update rates for a comfortable visual effect (Figure 9).

\subsection{Augmented Research Environment}

In [Gerv96] and [Szal97] we described "Studierstube", an architecture for multi-user augmented reality, with applications in visualization, presentation and education. Three dimensional graphics is presented to a work group, each of them wearing magnetically tracked seethrough HMDs. Every user can choose his or her viewpoint and configure the displayed image completely independent from all others. Models can have interleaved levels of information, which can be individually turned on and off by every participant without affecting the others' display. Co-operative work of researchers from different areas is thus supported without overloading the visualization.

\subsection{Augmented Scientific Visualization}

As our first major application for "Studierstube" we are currently developing a shared multi-user augmented research environment for scientific

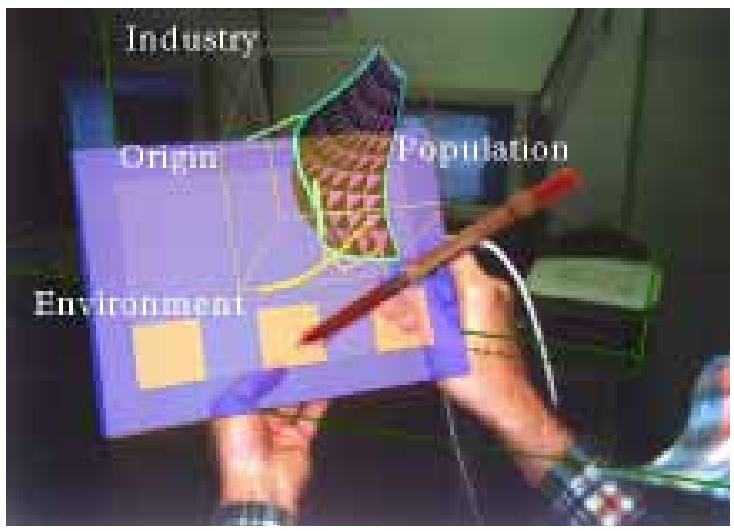

Figure 10. The "Wonderland” model [Gröl95] 
visualization, where we intend to make extensive use of the Personal Interaction Panel. Gröller, Wegenkittl [Grö195] and Löffelmann [Löff96] work in our lab on the visualization and investigation of non-linear dynamic systems. In a co-operation we work on the visualization of stream surfaces, trajectories and local behaviour of these systems in augmented environments. "Wonderland" is a four dimensional econometric model, which describes interactions between population growth, economic activity and environmental implications. Figure 10 shows a model of this dynamic system on the PIP while performing interaction with augmented buttons projected on the panels surface.

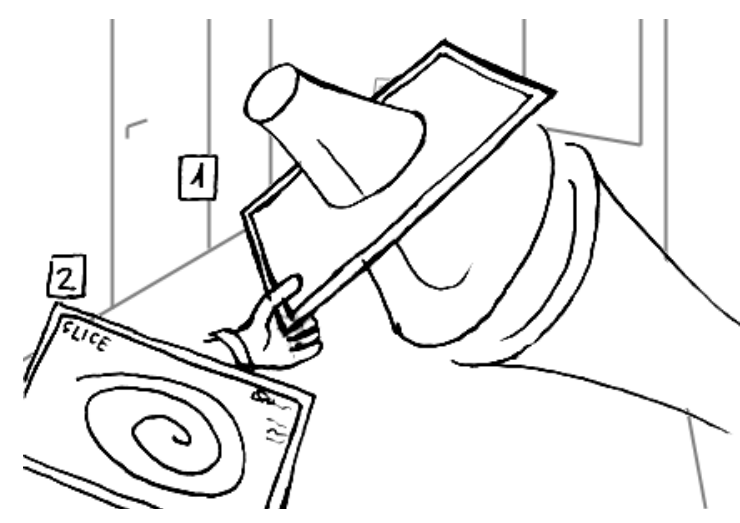

Figure 11. For scientific visualization the PIP can be used to specify and edit cutting planes ...

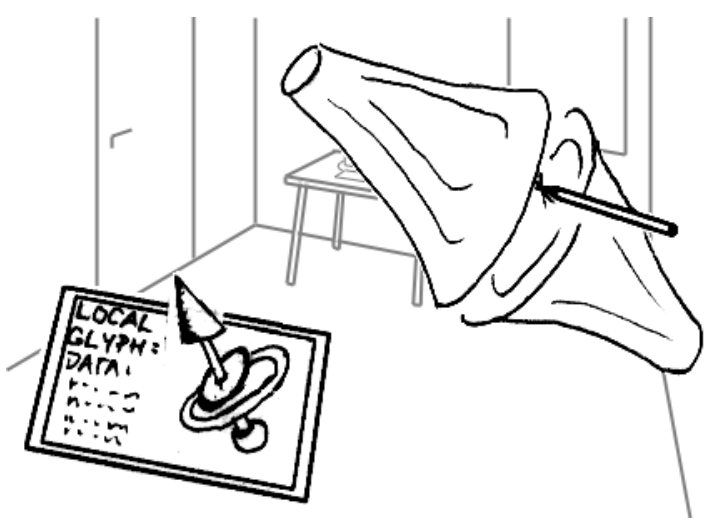

Figure 13. Multidimensional parameters at any point are shown using glyphs on the PIP or directly at the measuring point
In this application the PIP can be freely configured with special interaction metaphors for scientific visualization. In the simplest case steering parameters of the underlying simulation can be mapped easily onto the PIP and modified with the pen.

Other metaphors include defining 2D cross sections and $2 \mathrm{D}$ projections with the panel as shown in Figure 11. Representations can be displayed right on top of the PIP and exchanged among researchers directly or using projection walls. The augmented reality setup also allows the use of an additional high-resolution CRT monitor for the display of

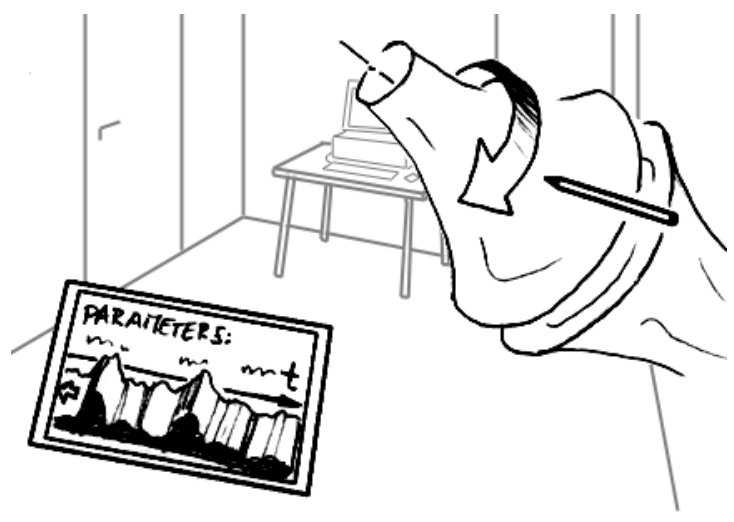

Figure 12. ... or measure simulated parameters at given locations and show instantly their evolution as 3D-graph on the panel

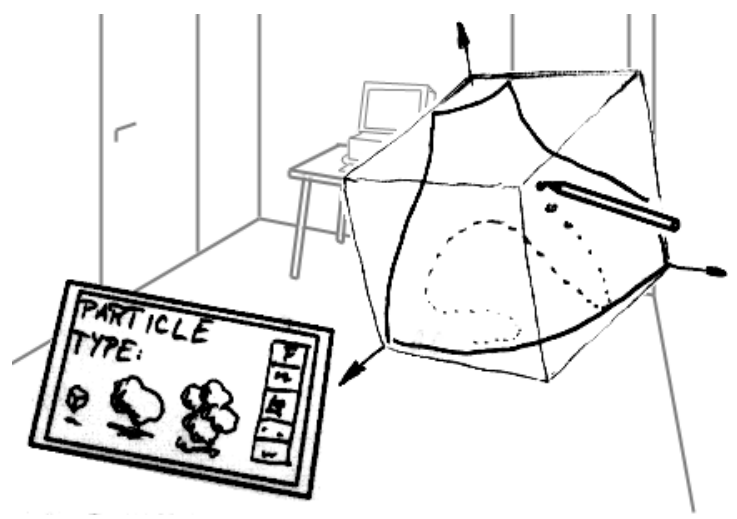

Figure 14. Introducing particles directly in an ongoing dynamic simulation should be very intuitive 
high quality 2D images (e.g., the mentioned cross sections) without leaving the augmented environment. Probing from certain points of the three dimensional representations include the display of temporal behaviour (Figure 12) and the representation of higher dimensional parameters by exact numerical data or graphical representations on the panel (Figure 13). In contrast to probing, where information is extracted from the model, the pen can also be used to specify the origin of particles introduced into the flow (Figure 14). With the PIP all these functions are supported by only one device, which fits to the actual needs by changing its appearance.

\section{Conclusion and Future Work}

We have introduced a multi-functional new 3D user interface, the Personal Interaction Panel, which consists of a magnetic tracked simple clipboard and pen, containing augmented information presented to the user by see-through HMDs. The low technical level the panel and pen itself allows flexible design of the interface and rapid prototyping. The natural two-handed interaction supported by this device makes the device fit to a rich variety of applications.

Initial evaluations have shown, that all test persons, including students who have never before experienced augmented or virtual reality, were familiar with the interface in a very short time, even if we missed to give a short introduction. Although they were surprised by the mixture of 2D and $3 \mathrm{D}$ display and interaction elements on the PIP, they did understand the interface. As they were used to work with both hands, they found the two-handed interface natural. Preliminary simple target acquisition tests have clearly shown, users targeting objects with the pen on the panel, held with the non-dominant hand, performed significant better than those trying to select objects with only the pen in one hand, even if the displayed image was not stereoscopic, so they had no additional depth cues.

However, the display technology we use is rather poor in quality and tracker cables are sometimes irritating, users like the interface, become familiar with it in a short time and did not report fatigue.
We will further improve the interface, especially for the scientific visualization domain and concentrate on a modular design to control arbitrary 3D applications. We incorporated the hand-drawn images to avoid confusion in the current diversity of our parallel test applications which are under instant development, some in very early stages. A uniform graphical redesign will help to generalize the appearance. Also an extensive user testing against other spatial input devices would be necessary to strengthen our claims we base on a set of users tried the first implementations of our interface up to this point.

\section{Acknowledgments}

This work has been supported by the Austrian Fond for Science and Research FWF Proj. No. P12074MAT and P11392-MAT. The authors would like to thank Dieter Schmalstieg for helpful discussions and Anton Fuhrmann providing some code for implementation.

\section{References}

[Amse95] D. Amselem: A Window on Shared Virtual Environments. Presence, Vol 4., No. 2, pp. 130-145 (1995)

[Baju92] M. Bajura, H. Fuchs, R. Ohbuchi: Merging Virtual Objects with the Real World: Seeing Ultrasound Imaginery within the Patient. Proceedings of SIGGRAPH'92, No. 2, pp. 203-210 (1992)

[Baju95] M. Bajura, U. Neumann: Dynamic Registration Correction in AugmentedReality Systems. Proceedings of the Virtual Reality Annual Symposium VRAIS'95, pp. 189-197 (1995)

[Bier93] E. Bier, M. Stone, K. Pier, W. Buxton, T. DeRose: Toolglass and Magic Lenses: The See-through Interface. Proceedings of SIGGRAPH'93, pp. 73-80, (1993)

[Butt92] J. Butterworth, A. Davidson, S. Hench: 3DM - A Three Dimensional Modeler Using a Head-Mounted Display. Proceedings of the Symposium on 
Interactive 3D Graphics 25, 2, pp. 135138 (1992)

[Conn92] D.B. Conner, S.S. Snibbe, K.P. Herndon, D.C. Robbins, R.C. Zeleznik, A. van Dam: Three-Dimensional Widgets. Proceedings of the Symposium on Interactive 3D Graphics, Vol. 25., No.2, pp. 183-188 (1992)

[Edwa97] J. Edwards, C. Hand: MaPS: Movement and Planning Support for Navigation in an Immersive VRML Browser. Proceedings of VRML'97, pp. 67-75 (1997)

[Frit93] G.W. Fritzmaurice: Situated Information Spaces and Spatially Aware Palmtop Computers. Communications of the ACM, Vol. 36, No. 7, pp. 39-49 (1993)

[Gerv96] M. Gervautz, D. Schmalstieg, Zs. Szalavári, K. Karner, F. Madritsch, A. Pinz: "Studierstube" - A Multi-User Augmented Reality Environment for Visualisation and Education. Technical Report TR-186-2-96-10, (ftp://ftp.cg. tuwien.ac.at/pub/TR/96/TR-186-2-96-10 Paper.ps.gz) (1996)

[Gob195] J. Goble, K. Hinckley, R. Pausch, J. Snell, N. Kassel: Two-Handed Spatial Interface Tools for Neurosurgical Planning. IEEE Computer, Vol. 28, No. 7, pp. 20-26 (1995)

[Grö195] E. Gröller, R. Wegenkittl, A. Milik, A. Prskawetz, G. Feichtinger, W.C. Sanderson: The Geometry of Wonderland. Chaos, Solitons \& Fractals, Vol. 7., No. 12, pp. 1989-2006 (1996)

[Guia87] Y. Guiard: Asymmetric Division of Labor in Human Skilled Bimanual Action: The Kinematic Chain as Model. Journal of Motor Behaviour, Vol. 19, No. 4, pp. 486-517 (1987)

[Harm96] R. Harmon, W. Patterson, W. Ribarsky, J. Bolter: The Virtual Annotation System. Proceedings of VRAIS '96, pp. 239-245 (1996)

[Hart96] J. Hartman, J. Wernecke: The VRML 2.0 Handbook: Building Moving Worlds on the Web. Addison-Wesley, New York (1996)

[Hinc94a] K. Hinckley, R. Pausch, J.C. Goble, N.F. Kassell: A Survey of Design Issues in Spatial Input. Proceedings of UIST'94, pp. 213-222 (1994)

[Hinc94b] K. Hinckley, R. Pausch, J.C. Goble, N.F. Kassell: Passive Real-World Interface Props for Neurosurgical Visualization. Proceedings of CHI '94 Conference on Human Factors in Computing Systems, pp. 452-458 (1994)

[Jaco93] R. H. Jacoby: Using virtual menus in a virtual environment. SIGGRAPH'93 Course, No. 43, pp. 7.1 - 7.8 (1993)

[Kabb94] P. Kabbash, W. Buxton, A. Sellen: Two-Handed Input in a Compound Task. Proceedings of CHI '94 Conference on Human Factors in Computing Systems, pp. 417-423 (1994)

[Krüg95] W. Krüger, C. Bohn, B. Fröhlich, H. Schüth, W. Strauss, G. Wesche: The Responsive Workbench: A Virtual Work Environment. IEEE Computer, Vol. 28, No. 7, pp. 42-48 (1995)

[Löff96] H. Löffelmann, L. Mroz, E. Gröller, W. Purgathofer: Streamarrows: Enhancing the Use of Streamsurfaces for the Visualization of Dynamical Systems. Accepted for publication in Visual Computer (1996)

[Mel88] B.W. Mel, S.M. Omohundro, A.D. Robinson, S.S. Skiena, K.H. Thearling, S. Wolfram, L.T. Young: Tablet: The Personal Computer in the Year 2000, Communications of the ACM, Vol. 31, No. 6, pp. 638-648 (1988)

[Paus95] R. Pausch, T. Burnette, D. Brockway, M. Weiblen: Navigation and Locomotion in Virtual Worlds via Flight into Hand-Held Miniatures. Proceedings of SIGGRAPH'95, pp. 399-401 (1995)

[Reki95] J. Rekimoto, K. Nagao: The World through the Computer: Computer Augmented Interactions with Real World 
Environments. Proceedings of UIST '95, pp. 29-36 (1995)

[Rose95] E. Rose, D. Breen, K.H. Ahlers, C. Crampton, M. Tuceryan, R. Whitaker, D. Greer: Annotating Real-World Objects Using Augmented Reality. Computer Graphics Developments in Virtual Environments, pp. 357-370 (1995)

[Sach91] E. Sachs, A. Roberts, D. Stoops: 3Draw: A Tool for Designing 3D Shapes. IEEE Computer Graphics \& Applications, Vol. 11, No. 6, pp. 18-26 (1991)

[Serr95] L. Serra, T. Poston, N. Hern, C.B. Choon, J.A. Waterworth: Interaction Techniques for a Virtual Workspace. Proceedings of VRST'95 also as online document at http://ciemed.iss.nus.sg/ research/3dinterfaces/papers/vrst95/vrst95 $-1 . h t m l(1995)$

[Szal97] Zs. Szalavári, D. Schmalstieg, A. Fuhrmann, M. Gervautz: "Studierstube"-
An Environment for Collaboration in Augmented Reality. To be published in Journal of Virtual Reality also Technical Report TR-186-2-97-01 (ftp://ftp.cg.tuwien.ac.at/pub/TR/97/TR186-2-97-01Paper.ps.gz) (1997)

[Stat96] A. State, G. Hirota, D. Chen, W. Garrett, M. Livingston: Superior Augmented Reality Tracking by Integrating Landmark Tracking and Magnetic Tracking. Proceedings of SIGGRAPH'96, pp. 429-438 (1996)

[Stoa95] R. Stoakley, M.J. Conway, R. Pausch: Virtual Reality on a WIM: Interactive Worlds in Miniature. Proceedings of CHI'95 Conference on Human Factors in Computing Systems (1995)

[Weis91] M. Weiser: The Computer for the 21st Century. Scientific American, Vol. 265, No. 3, pp. 94-104 (1991)

[Wlok95] M.M. Wloka, E. Greenfield: The Virtual Tricoder: A Uniform Interface for Virtual Reality. Proceedings of UIST '95, pp. 39-40 (1995) 